\title{
Shock Capturing Anomalies and the Jump Conditions in One Dimension
}

\author{
Daniel W. Zaide* and Philip L. Roe ${ }^{\dagger}$ \\ Department of Aerospace Engineering, University of Michigan, Ann Arbor, MI 48109
}

\begin{abstract}
In this work we examine how the nonlinearity of the Rankine-Hugoniot jump conditions dictates the behavior of shock capturing methods, particularly of Godunov-type schemes. Here we present four related onedimensional examples of the artifacts caused by this: sub-cell shock position in the stationary shock, the slowly moving shock, the wall heating problem, and the carbuncle phenomenon. Each one of these well known problems is shown to be directly related to the nonlinearity of the Hugoniot and numerical experiments are performed to verify the connection. Lastly, a system with a straight Hugoniot is described and shown not to suffer from any of these phenomena.
\end{abstract}

\section{Introduction}

Why do numerical shocks jump to the wrong conclusions? When a physical shockwave is formed, it moves through the flow with a certain speed, having some finite width determined by physical dissipation until it encounters some event in its path. For numerical shockwaves, however, a numerical width is enforced, often much greater than the physical width. With this numerical width comes the formation of intermediate states having no direct physical interpretation. These 'filler' states serve the same purpose as the filler within a rough stone wall - we are only interested on how it looks from either side, and we just put anything within it, as long as the final product is acceptable. Of course, as the mesh is refined, these intermediate states do not go away; they simply occupy less space. Their existence does not compromise the Lax-Wendroff Theorem ${ }^{1}$ that consistency and conservation, taken together, imply convergence to a weak solution. Convergence is only to be expected in an integral sense. The existence of intermediate states does raise some doubt, however, about how closely a captured shockwave may emulate an ideal discontinuous shockwave, or a real physical one.

There are in fact several types of error associated with captured shockwaves, and their impact on the quality of the solution ranges from irritating to calamitous. In this paper we review four of these errors, and link them all to a common cause. Our investigations are limited to the one-dimensional case. One error is the ambiguity concerning the location

\footnotetext{
${ }^{*}$ Graduate Student, AIAA Member
}

${ }^{\dagger}$ Professor, AIAA Fellow 
of a captured shock. Another is the creation of persistent "entropy traces" caused by an inappropriate production of entropy, which then propagates in a physical manner. One such example, the wall heating phenomenon exemplified by Noh's ${ }^{2,3}$ well known test problem has caused much grief, both in the Eulerian and Lagrangian communities. A special case of this is the so-called "start-up error". A third problem is that of the slowly moving shockwave. ${ }^{4-6}$ Finally we consider the "carbuncle" $\cdot^{7-9}$ Although this is really a multidimensional phenomenon, it does have a one-dimensional manifestation.

It has been previously postulated by Barth ${ }^{10}$ and Robinet et al. ${ }^{11}$ among others that many of these numerical problems are related, often applying a linearized analysis to demonstrate the similarity. In this work, we apply a different approach to study these problems, choosing to link them through the nonlinearity of the Hugoniot curve.

\section{Governing Equations}

While this work only examines this issue for the Euler equations with a gamma-law equation of state, similar work ${ }^{5}$ suggests that these problems are found in other, related systems. The Euler equations, here shown in one dimension, are

$$
\mathbf{u}_{t}+\mathbf{f}(\mathbf{u})_{x}=0
$$

expanded as

$$
\frac{\partial}{\partial t}\left[\begin{array}{c}
\rho \\
\rho u \\
E
\end{array}\right]+\frac{\partial}{\partial x}\left[\begin{array}{c}
\rho u \\
\rho u^{2}+p \\
u(E+p)
\end{array}\right]=\mathbf{0}
$$

with the equation of state $p=p(\rho, i)$. For an ideal gas this is

$$
p=(\gamma-1) \rho i, \quad \rho i=\left(E-\frac{1}{2} \rho u^{2}\right) .
$$

The speed of sound is then $a=\sqrt{\left.\frac{\partial p}{\partial \rho}\right|_{s}}=\sqrt{\frac{\gamma p}{\rho}}$.

\section{II.A. The Rankine-Hugoniot Jump Conditions}

For a shockwave moving in one dimension with speed $S$ the jump conditions are given by

$$
[\mathbf{f}]=S[\mathbf{u}] .
$$

Suppose that the left (preshock) state is completely known, as $\rho_{L}, u_{L}, p_{L}$. If the density on the right is prescribed to be $\rho_{R}$, then the remaining postshock variables are given by ${ }^{12}$

$$
\begin{aligned}
\frac{p_{R}}{p_{L}} & =\frac{(\gamma+1) \rho_{R}-(\gamma-1) \rho_{L}}{(\gamma+1) \rho_{L}-(\gamma-1) \rho_{R}} \\
u_{R}-u_{L} & =\left(p_{L}-p_{R}\right) \sqrt{\frac{2}{\rho_{L}\left((\gamma-1) p_{L}+(\gamma+1) p_{R}\right)}}
\end{aligned}
$$

The curve traced out in phase space by solving these equations is the Hugoniot curve. It has two branches. If we take $\rho_{R}>\rho_{L}$ then each point on the curve represents a valid state 
behind a (generally moving) shockwave, and we will refer to this as the physical branch of the Hugoniot curve. If we take $\rho_{R}<\rho_{L}$ this generates the nonphysical branch of the Hugoniot. A jump from $\mathbf{u}_{L}$ to $\mathbf{u}_{R}$ would violate thermodynamics. Note however, that each point on the nonphysical branch represents a state from which $\mathbf{u}_{L}$ could be reached by a valid shockwave.

\section{The Shock Capturing Method}

We study our four problems through a first-order in space and time shock capturing scheme of the form

$$
\mathbf{u}_{j}^{n+1}=\mathbf{u}_{j}^{n}-\frac{\Delta t}{\Delta x}\left(\mathbf{f}_{j+\frac{1}{2}}^{n}-\mathbf{f}_{j-\frac{1}{2}}^{n}\right)
$$

where the interface flux, $\mathbf{f}_{j+\frac{1}{2}}=\mathbf{f}\left(\mathbf{u}_{j}, \mathbf{u}_{j+1}\right)$, is computed using either the exact Riemann solver or Roe's approximate Riemann solver. ${ }^{13}$ These schemes are interesting because they attempt to employ physical reasoning, which appears to be successful in some respects (crisply captured discontinuities) but less so in others, notably the four issues raised above. Also, they have certain properties that encourage analysis. We restrict ourselves to firstorder accuracy partly for simplicity, but also because experience suggests that high-order schemes suffer all of the problems that afflict their first-order versions, merely providing better resolution of the spurious physics. ${ }^{14}$

\section{III.A. Intermediate Shock States}

As already noted, captured shocks contain intermediate states, as seen in Figure 1, that are are not part of the exact solution but are required for the discrete representation. While the number of required intermediate states varies from scheme to scheme and depends on the Courant number and Riemann solver, ${ }^{4,15}$ among other things, all conservative schemes produce them.
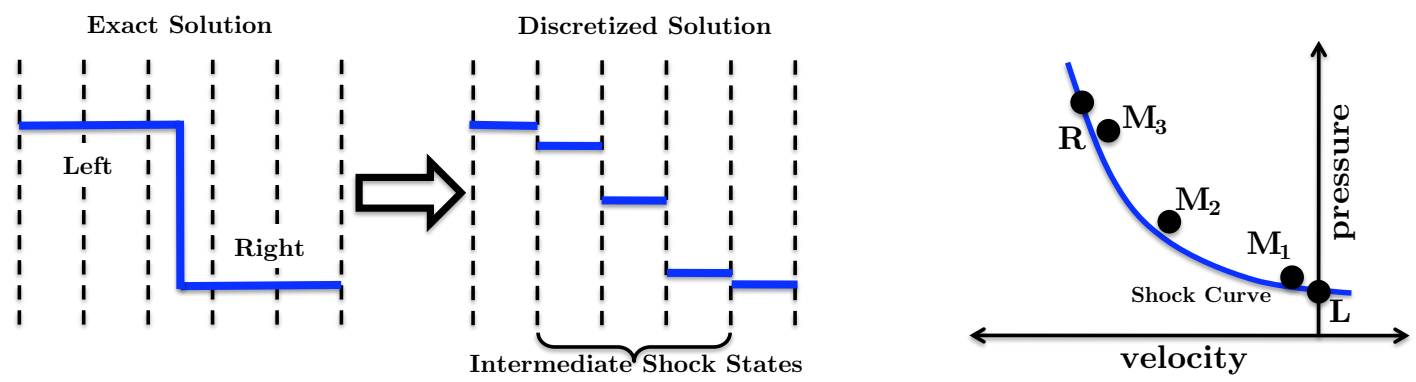

Figure 1. Representative single shock problem. (left) Exact Solution. (middle) Discretized exact solution for a single shockwave with resulting intermediate states.(right) Shock curve, and locations of intermediate states.

One might imagine that the set of intermediate states could all lie along the Hugoniot curve, but this not possible. Suppose that states L and R are connected through an intermediate state $M$ that lies on the physical Hugoniot that passes through L. There cannot be another shock transition from $\mathrm{M}$ to $\mathrm{R}$, because $\mathrm{M}$ does not lie on the nonphysical Hugoniot from $\mathrm{R}$. Therefore the transition from $\mathrm{M}$ to $\mathrm{R}$ would have to involve waves of the other families. 
For the special case of a stationary shockwave, calculated either the exact or Roeaveraged flux, a transition with one intermediate state is in fact possible. ${ }^{16,17}$ The compression from $\mathrm{M}$ to $\mathrm{R}$ is accomplished by a physical shock of the $u-a$ family. This ensures that no right-going wave is generated at the interface MR. The state at the interface LM turns out to be supersonic, so no left-going waves are generated at LM. All of the waves generated within the shock region remain trapped. The set of possible states $\mathrm{M}$ lie on the nonphysical Hugoniot through $\mathrm{R}$ as shown in Figure 2 .

Of note is that, wherever these intermediate states lie, they are always assumed to satisfy local thermal equilibrium in a cell-averaged sense, and therefore to obey the equilibrium equation of state (3). However, from the exact solution to the Navier-Stokes equations, we know that local thermal equilbrium does not hold inside the shock itself. This suggests a form of 'interpretation' error, an error made in the philosophical approach we take when assigning meaning to the shock-captured solution.

\section{Ambiguity in Stationary Shock Position}

To properly understand the ambiguity of shock position for a stationary shock, we must first decide how we would like to calculate this position. For a scalar conservation law, the shock position can be determined using the equal-area rule ${ }^{18}$ within a control volume around the shock. As illustrated in Figure 3, the equal-area rule states that the shock position divides the discrete solution into two regions such that the integrated error in each region has the same absolute value. This comes directly from conservation principles and provides an unamiguous location for a shock that separates two uniform states.

One would hope that for a system of conservation laws a similar rule could be used to determine the shock position from any of the conserved variables, and that these shock positions would agree. In fact, there is systematic disagreement. This is already known, although we are not aware of any formal publication on the matter, and the fact does not seem to be well-known. The discrepancy can be proved for the special case of a stationary shock, and can be a significant fraction of a cell-width.

It was shown above that a one-dimensional Euler code employing either the exact or Roe-averaged Riemann solver as a flux function has solutions of the following form. Left and right states satisfying the conditions for a stationary jump are separated by a single intermediate state that lies on the nonphysical Hugoniot of the right state. 

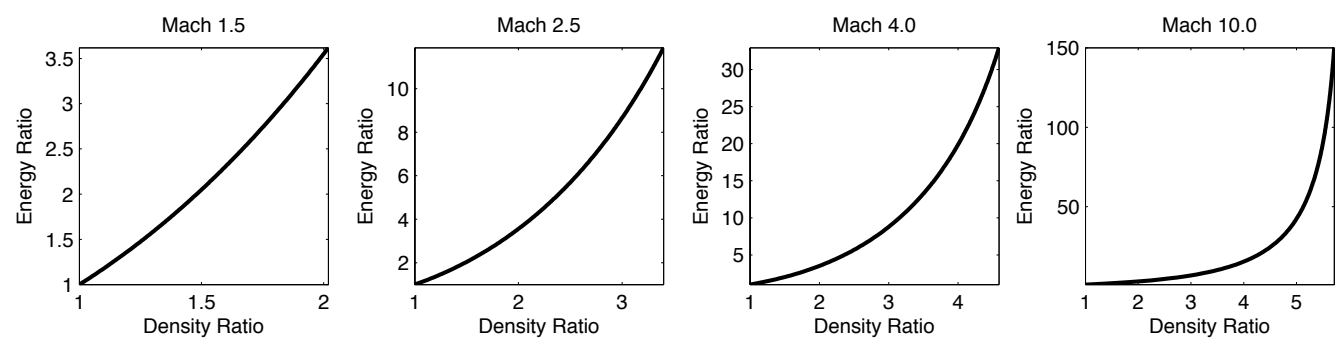

Figure 4. Loci of density versus energy ratios in the intermediate cell for initial Mach numbers of $1.5,2.5,4.0$ and 10.0.

The locus of all possible states $\mathbf{u}_{M}$ can therefore be computed using standard shock relationships. We would obtain shock locations of

$$
x_{S}(\rho)=\frac{\rho_{M}-\rho_{R}}{\rho_{L}-\rho_{R}} \quad x_{S}(E)=\frac{E_{M}-E_{R}}{E_{L}-E_{R}}
$$

by applying conservation of density or energy respectively. We would hope that these would be the same. Plots of $E_{M} / E_{R}$ versus $\rho_{M} / \rho_{R}$ are shown in Figure 4, and under suitable scaling these are plots of $x_{S}(E)$ versus $x_{S}(\rho)$. The two locations are roughly equal for a weak shock but become increasingly nonlinear as Mach number increases. Therefore the two predictions do not agree. At Mach 10, the maximum discrepancy is about $\Delta x / 2$. The situation is even worse if we try to predict the shock position using momentum, because this is equal for the left and right states, but finite for the intermediate state. See Barth ${ }^{10}$ for commentary on this point.

We have found in experiments with other shock-capturing schemes that there is almost always a discrepancy in the location of a captured shock, although when the number of intermediate states increases the discrepancy tends to be smaller.

\section{The Wall Heating Phenomenon}

A notorious problem for those who compute high speed flow is the Noh problem, ${ }^{2}$ a seemingly trivial Riemann problem with initial data corresponding to the collision of two equal shocks, or equivalently the reflection of a single shock from a solid wall. The initial data is $\mathbf{u}_{0}=\left(\rho_{0}, \rho_{0} u_{0}, E_{0}\right)^{T}$ but can be reduced to a one parameter family with $\rho_{0}=1, u_{0}=-1$ and Mach number, $M_{0}=\frac{1}{\sqrt{\gamma p_{0}}}$, as a free parameter. Virtually all shock-capturing methods provide quite good solutions for pressure and velocity, but
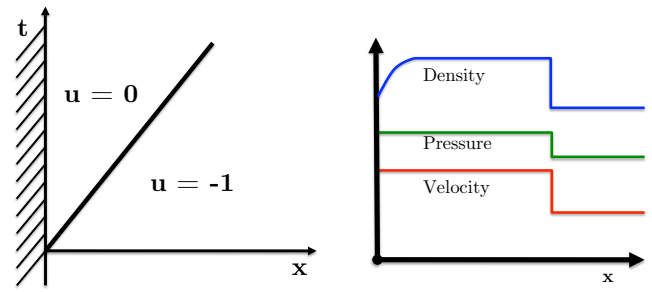

Figure 5. (left) Space-time representation of the Noh problem. (right) Representative solutions for the Noh problem after the shock has left the wall area. Note the density defect at the wall. predict too small a density in a small region at the origin ${ }^{\mathrm{a}}$, as in Figure 5. In consequence the temperature there is too high, so that this

\footnotetext{
${ }^{a}$ The difficulty is exacerbated by posing the problem in cylindrical or spherical geometry, but still occurs in one dimension.
} 
and related phenomena have been called wall heating. In the more than twenty years since Noh proposed the problem, no satisfactory solution has been exhibited that would carry over to other settings, nor is there even any generally accepted explanation of the mechanism. Our demonstration is with $\gamma=5 / 3$.

We solve the Noh problem, and then try to locate the shock by considering the mass, momentum or energy in a control volume. In fact, two control volumes are used:

1. Local - contains only the region immediately around the shock.

2. Global - contains the whole domain, including the density defect in the calculation.

Figures 6 and 7 show results for Mach 1.1 and $10^{6}$ shocks, calculated with a CFL number of 0.5. On the left, the complete solution for conserved variables is shown. In the middle, shock positions are calculated using a local control volume. All three conserved quantities produce different shock positions. The position calculated from the energy distribution agrees almost perfectly with the exact solution, but the position calculated from density is about $0.2 \Delta x$ ahead of it. This can be interpreted as meaning that the shock contains excess mass.

However, on the right, shock positions have been calculated using the global control volume. Here, the shock positions computed using density and energy are equal, showing that the density defect observed at the wall exactly compensates the excess in the shock. With hindsight, this should have been expected. Conservation ensures that all of the mass that should be present really is present. Therefore, if it is deficient in one place it must be excessive in another. It seems possible that if the ambiguity in shock position could be resolved, there might be no wall heating.

Further studies on this problem lead to two key observations:
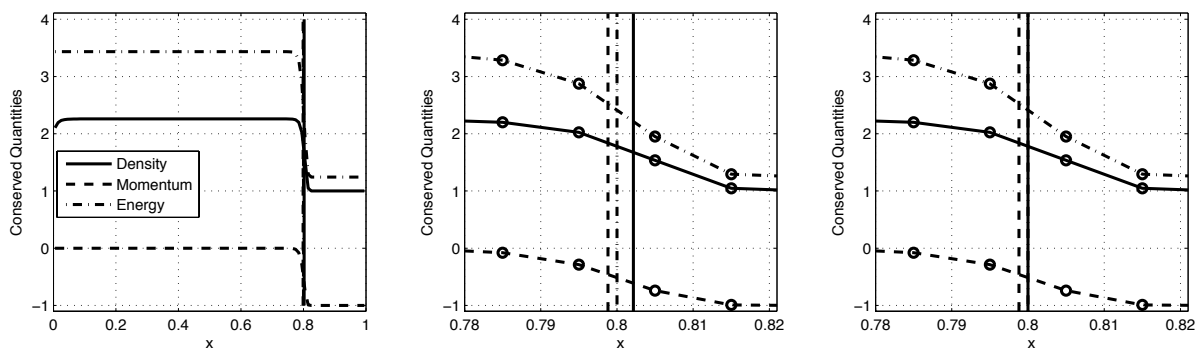

Figure 6. Conserved quantities for a Mach 1.1 shock. (left) Full solution. (middle) Sub-cell shock locations computed using a local control volume. (right) Sub-cell shock locations computed using a global control volume.
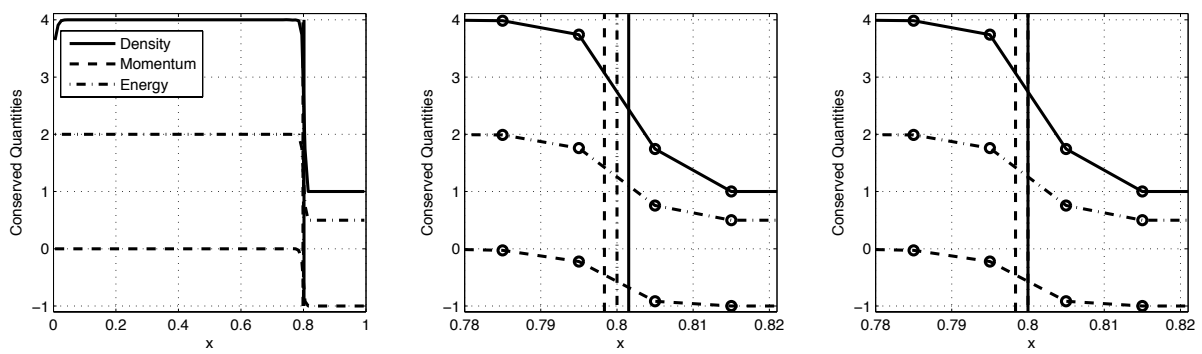

Figure 7. Conserved quantities for a Mach $10^{6}$ shock. Same three plots as above. 
- The distance between shock positions computed by any two conserved variables is constant with time after the shock has settled.

- The distance between shock positions computed by any two conserved variables is fairly independent of Mach number.

These observations demonstrate that despite the difference in sub-cell shock position, the shock speed in each conserved variable is the same and that while the shock strength may vary, the ambiguity remains the same and is directly compensated by the error at the wall. In other situations the 'missing mass' may appear somewhere else, but it will have to appear somewhere. The second observation would appear to contradict the theory in Section IV, however in the Noh problem, the shock now consists of multiple intermediate states, especially at the lower Mach numbers, and the theory for a single shock state does not apply.

\section{The Slowly Moving Shockwave Phenomenon}

Another known example of a numerical anomaly is the slowly moving shockwave phenomenon. Examined in detail by Roberts, ${ }^{4}$ Arora and Roe ${ }^{5}$ and by Karni and Canic ${ }^{6}$ among others, the observation is that spurious waves are thrown off by slowly moving shocks. These waves are purely numerical, but once created, they will be propagated as though they were real. The cause of this phenomenon is that not all of waves produced by solving Riemann problems involving the intermediate states are of the same family as the shockwave.

To show that nonlinearity is directly responsible for the spurious waves, we conducted experiments involving small perturbations of the stationary shock. This limiting case will be produced by prescribing the left state to be $\left[\rho_{L}, u_{L}, p_{L}\right]^{T}=\left[1,1,1 / \gamma M_{L}^{2}\right]^{T}$ with the right state

$$
\begin{aligned}
\rho_{R} & =\frac{(\gamma+1) M_{L}^{2}}{(\gamma-1) M_{L}^{2}+2}, \\
u_{R} & =\frac{1}{\rho_{R}}=\frac{(\gamma-1) M_{L}^{2}+2}{(\gamma+1) M_{L}^{2}}, \\
p_{R} & =\frac{1}{\gamma M_{L}^{2}}\left(1+\frac{2 \gamma}{\gamma+1}\left(M_{L}^{2}-1\right)\right) .
\end{aligned}
$$
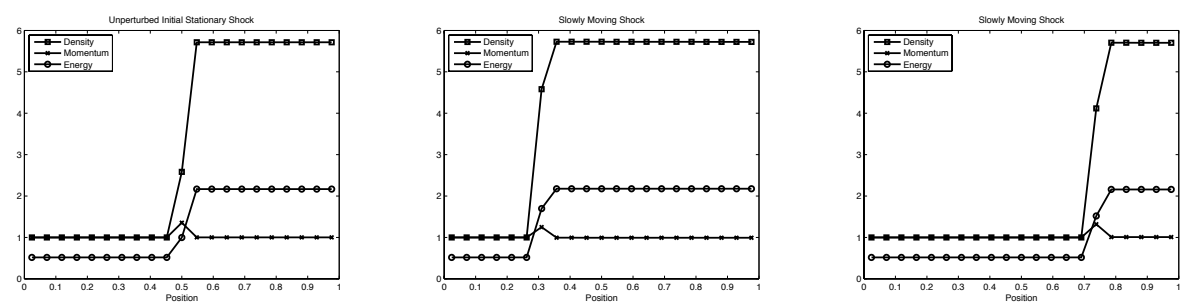

Figure 8. (left) An initially stationary Mach 10 shockwave. (middle) A $1 \%$ perturbation to create a slowly moving shock moving to the left. (right) A $1 \%$ perturbation to create a slowly moving shock moving to the right. 

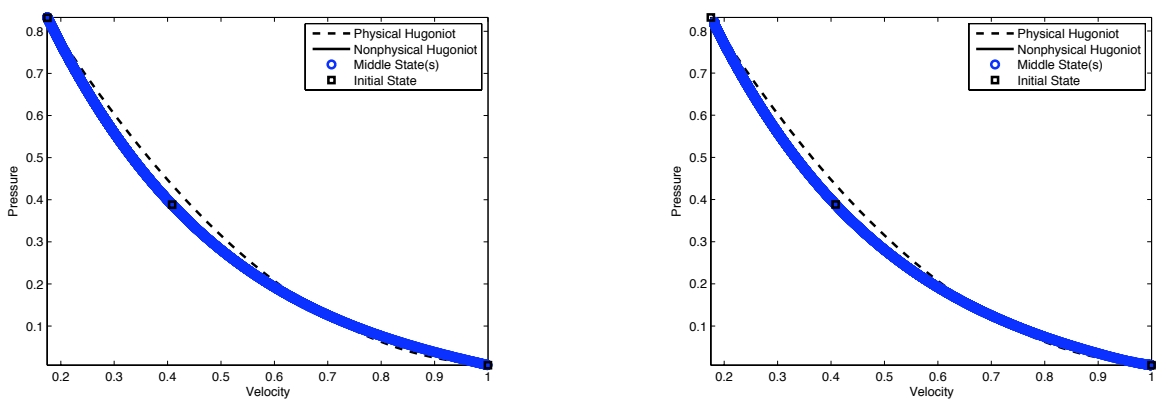

Figure 9. Intermediate States from the Slowly Moving Shock. While the intermediate states do not lie exactly on the nonphysical Hugoniot, they do lie slightly off of it, on curve corresponding to two other end states. On the left, the intermediate states correspond to the left slowly moving shock. On the right, they correspond to the right moving shock.

By slightly perturbing this right state, slowly moving shocks in either direction can be created. Figure 8 shows two equal and opposite perturbations of a Mach 10 stationary shockwave.

In a previous investigation by Arora and Roe ${ }^{5}$ plots were made in phase space showing every state that appears in the solution at any time step. This reveals the locus that is followed by the intermediate states. Some of the loci presented in ${ }^{5}$ were highly intricate. We tested here shockwaves that moved even more slowly, to check that approaching the limit of zero speed caused the locus to approach the steady equilibrium locus. In other words, we wanted to see if the slowly moving shock was a regular or singular perturbation of a stationary shock. We found that the perturbation was usually regular, with exceptions discussed in the next section, and which are ignored here.

We find, then, that each snapshot of a slowly moving shock can be viewed very closely as a sample of the stationary cases. Figure 9, shows that the locus for a shock moving very slowly to the left is the same as that for a shock moving very slowly to the right.

To see the connection with the curved Hugoniot, consider that as the shock moves across the grid, the amount of discrepancy between its positions, as calculated from each conserved variable, will change periodically. The discrepancy will repeat itself every time that the shock has crossed one more cell. If we interpret this as meaning that the shock has excess mass that varies with the same period, there must be, by conservation, a mass deficit elsewhere. This accounts for the periodic shedding of the spurious waves.

\section{The Carbuncle Phenomenon}

Often in hypersonic flow computations, regions of anomalous shock structure occur, generically called "carbuncles". A related, and probably relevant, behavior can be seen in one dimension. As analysed by Barth, ${ }^{10}$ Serre, ${ }^{19}$ and Dumbser et al. ${ }^{20}$ not all of the equilibrium solutions (involving an intermediate state lying on the nonphysical Hugoniot through $\mathrm{R}$ ) are stable. The attempt to compute a stationary shock at a position corresponding to one of these unstable equilibria results in a shock that does not remain stationary. Either the shock moves to a stable location, or, under suitable boundary conditions that exactly conserve mass, it enters into a limit cycle. That cycle involves the shedding of spurious waves and their reflection from the downstream boundary. These unstable equilibria only exist at 
high Mach number. The critical value for $\gamma=7 / 5$ is about 6.0 .

As in Kitamura, Roe, and Ismail ${ }^{7}$ and Chauvat, Moschetta and Gressier $^{8}$ we specify the intermediate point by a parameter $x_{S}$ such that $\rho_{M}=x_{S} \rho_{L}+\left(1-x_{S}\right) \rho_{R}$. We also examine cases where the initial intermediate state lies off of either Hugoniot to observe its behavior. Thus, we examine cases where the initial data corresponds to either a stable or an unstable location, and the initial profile does or does not represent an equilibrium solution. In Figure 10, four cases are shown.
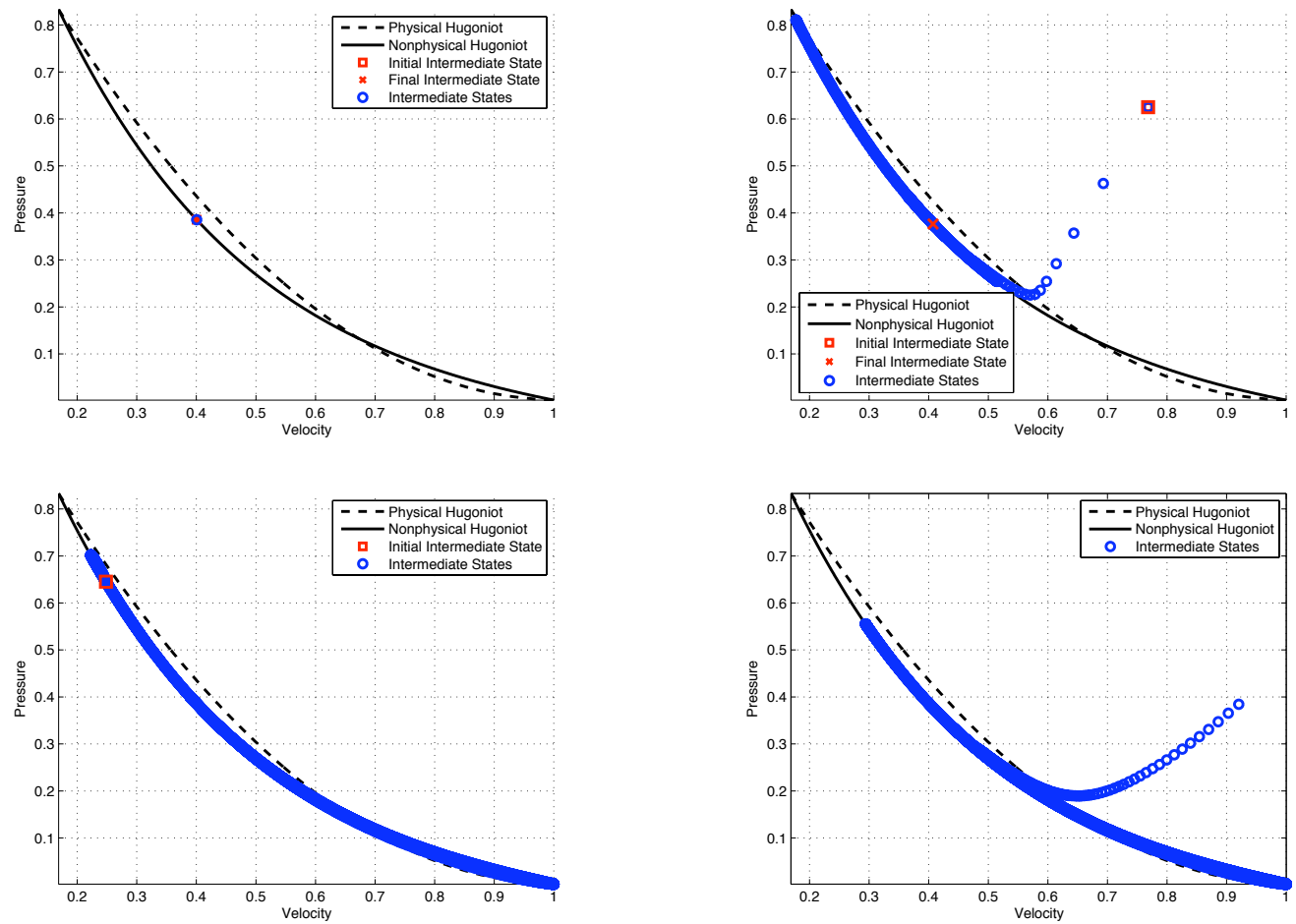

Figure 10. All results shown with Roe's Riemann solver, and a Mach 20 shock. (top) Two carbuncle free cases. On the left, $x_{S}=0.5$ and the initial intermediate state lies on the nonphysical Hugoniot. On the right, the initial intermediate state is off both Hugoniot curves.(bottom) Two cases with carbuncles. On the left, $x_{S}=8.0$. On the right, the initial intermediate state is again off both Hugoniot curves.

Stable location, equilibrium data. As would be expected, the code exactly preserves the initial data.

Stable location, nonequilibrium data. The locus of intermediate states moves directly to the equilibrium locus and then travels along it to find the equilibrium that conserves mass.

Unstable location, equilibrium data. The intermediate states "hunt" back and forth along the equilibrium curve, without finding a stable location. The outflow boundary condition was here specified to be constant mass flow, so there is no way that the code can shed surplus mass.

Unstable location, nonequilibrium data. The intermediate states track quickly onto the equilibrium locus, but are not able to find a stable location. 
In those cases where no stable equilibrium exists, examining intermediate states reveals that all lie on the nonphysical Hugoniot curve, identical to those seen in Figure 9 for a left and right moving shockwave. Viewing the carbuncle in this frame, it is observed that in one dimension, it is no more than a slowly moving shock that is unable to find a stable position, moving in one direction and then the other within the cell. Since the slowly moving shock phenomenon is due to the nonlinearity of the Hugoniot curve, the connection between the carbuncle and this nonlinearity is made. Kitamura, ${ }^{7}$ however, showed that some shocks were unstable in $2 \mathrm{D}$ even though stable in $1 \mathrm{D}$, so this cannot be a complete explanation.

\section{A Linear Hugoniot?}

If much of the odd behavior of captured shocks is associated with curvature of the Hugoniot, it is interesting to ask the question, 'what if the Hugoniot were linear?'. This is mathematically possible, even if the conservation laws are nonlinear. In fact, there is a substantial literature on "straight line systems", for example Temple ${ }^{21}$ and Bressan and Jensen. $^{22}$ Arora and Roe ${ }^{5}$ claimed that $2 \times 2$ systems with linear Hugoniots do not suffer from the slowly moving shock phenomenon. Here, we examine a $3 \times 3$ system used as a model of magnetohydrodynamics by Myong and Roe. ${ }^{23}$

$$
\left[\begin{array}{l}
u \\
a \\
b
\end{array}\right]_{t}+\left[\begin{array}{c}
\frac{1}{2}\left(u^{2}+a^{2}+b^{2}\right) \\
u a \\
u b
\end{array}\right]_{x}=0
$$

This system has a propagation speed, $c=\sqrt{a^{2}+b^{2}}$ (which is also a conserved quantity) and supports genuinely nonlinear "acoustic waves" that move with speeds $u \pm c=$ constant and a contact discontinuity with speed $u$ (and constant $u$ and $c$ across it). Since both the physical and nonphysical Hugoniot are linear and identical, we would expect that there would be agreement in shock position and that there would be no evidence of any of the aforementioned phenomena. Indeed this is the case. Only results for the Noh problem and
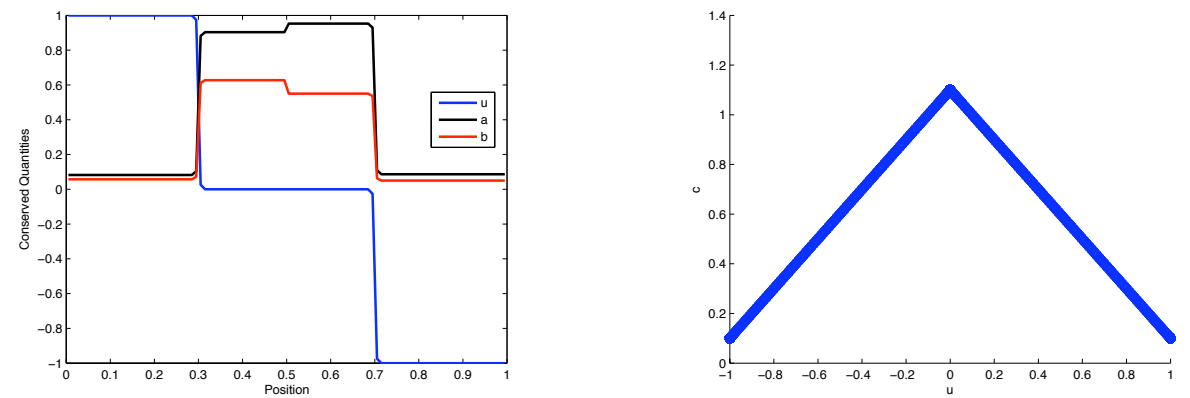

Figure 11. The Noh problem for the system of equations 12 with Mach number 10. On the left, the solution after the reflecting shocks have moved sufficiently far away from the wall. While there are jumps in $a$ and $b$ across the contact discontinuity, there is no remaining error. On the right, all solution states are plotted in $c$ vs $u$ space. All states lie on either Hugoniot curve.

the slowly moving shock are shown, since there is no ambiguity in stationary shock position and an exhaustive numerical study of carbuncle-like issues reveals no evidence of any similar problem. For the Noh problem, we can construct an analogous symmetric problem with two reflecting shockwaves separated by a stationary contact discontinuity. In Figure 11, there is 

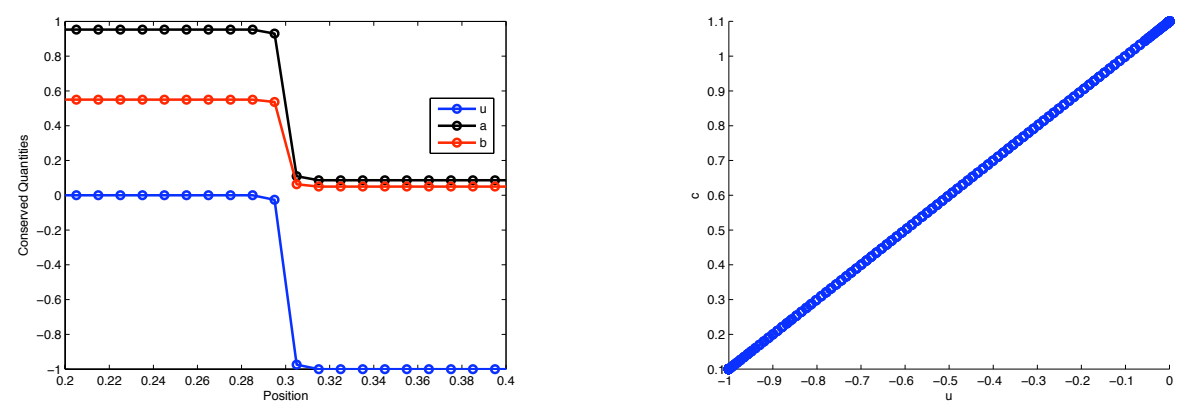

Figure 12. The Slowly moving shock problem for Equations 12 with Mach number 10. On the left, the solution after the shock has passed through several cells. On the right, all solution states are plotted in $c$ vs $u$ space. All states lie on the correct Hugoniot curve.

no error made along the contact discontinuity, and thus no 'wall heating'-like behavior. In Figure 12, a single shock moves slowly through the domain. In both problems, there are no spurious waves shed, and all intermediate states lie on the Hugoniot. While this system does not prove that nonlinearity is the sole cause of all of these issues, it suggests that correct placement of intermediate states may result in better solutions and the alleviation of many numerical shockwave anomalies.

\section{Conclusions and Future Work}

In this paper, we have established connections between four common defects of shockcapturing methods, by linking each of them to the nonlinearity of the Hugoniot curve. Since that nonlinearity is a physical fact, it would seem that an over-reliance on physical derivations may have some drawbacks, especially if they rely on assumptions of thermodynamic equilibrium. At present, the benefits of schemes that crisply resolve discontinuities seem to outweigh the drawbacks, but at the cost of various fixes and workarounds.

At the present time we can only speculate about the possibility of a completely satisfactory method, but almost certainly it will involve giving up the property of capturing shocks with only one intermediate state. However, for Navier-Stokes applications, it will important to retain the ability to recognise contact discontinuities. We are currently experimenting with various ways of enforcing a better-behaved set of intermediate states. We have considered insisting on a fixed width for the shock, and also allowing cells that are not in local thermodynamic equilibrium. Although both approaches show some promise, they are not yet ready for presentation.

Future work will focus on strengthening the connections made, by testing a wider range of Riemann solvers and examining the relationship of stationary shocks to their transient relatives. By instituting a form of shock structure control, we hope to position intermediate shock states to minimize the creation of spurious waves.

\section{Acknowledgements}

The first author is currently supported by the DOE NNSA under the Predictive Science Academic Alliances Program by grant DE-FC52-08NA28616 and by a Natural Sciences and Engineering Research Council of Canada (NSERC) Postgraduate Scholarship. 


\section{References}

${ }^{1}$ Lax, P. and Wendroff, B., "Systems of Conservation Laws," Communications on Pure and Applied Mathematics, Vol. 13, May 1960, pp. 217-237.

${ }^{2}$ Noh, W. F., "Errors for calculations of strong shocks using an artificial viscosity and an artificial heat flux," Journal of Computational Physics, Vol. 72, No. 1, 1987, pp. 78-120.

${ }^{3} \mathrm{Xu}, \mathrm{K}$. and $\mathrm{Hu}$, J., "Projection dynamics in Godunov-type schemes," Journal of Computational Physics, Vol. 142, No. 2, 1998, pp. 412-427.

${ }^{4}$ Roberts, T., "The behavior of flux difference splitting schemes near slowly moving shock waves," Journal of Computational Physics, Vol. 90, No. 1, 1990, pp. 141-160.

${ }^{5}$ Arora, M. and Roe, P. L., "On postshock oscillations due to shock capturing schemes in unsteady flows," Journal of Computational Physics, Vol. 130, No. 1, 1997, pp. 25-40.

${ }^{6}$ Karni, S. and Canic, S., "Computations of slowly moving shocks," Journal of Computational Physics, Vol. 136, No. 1, 1997, pp. 132-139.

${ }^{7}$ Kitamura, K., Roe, P., and Ismail, F., "Evaluation of Euler Fluxes for Hypersonic Flow Computations," AIAA Journal, Vol. 47, No. 1, 2008, pp. 44-53.

${ }^{8}$ Chauvat, Y., Moschetta, J., and Gressier, J., "Shock wave numerical structure and the carbuncle phenomenon," International Journal for Numerical Methods in Fluids, Vol. 47, No. 89, 2005, pp. 903-909.

${ }^{9}$ Quirk, J., "A contribution to the great Riemann solver debate," International Journal for Numerical Methods in Fluids, Vol. 18, No. 6, 1994, pp. 555-574.

${ }^{10}$ Barth, T., "Some notes on shock resolving flux functions. Part 1: Stationary characteristics," 1989.

${ }^{11}$ Robinet, J., Gressier, J., Casalis, G., and Moschetta, J., "Shock wave instability and the carbuncle phenomenon: same intrinsic origin?" Journal of Fluid Mechanics, Vol. 417, 2000, pp. 237-263.

${ }^{12}$ Courant, R. and Friedrichs, K., "Supersonic flow and shock waves," Interscience, Jan 1948.

${ }^{13}$ Roe, P., "Approximate Riemann solvers, parameter vectors, and difference schemes," Journal of Computational Physics, Jan 1981.

${ }^{14}$ Johnsen, E., Lele, S. K., and Larsson, J., "Analysis and Correction of Errors Generated by Slowly Moving Shocks," 49th AIAA Aerospace Sciences Meeting, Dec 2011, pp. 1-12.

${ }^{15}$ Osher, S. and Solomon, F., "Upwind difference schemes for hyperbolic systems of conservation laws," Mathematics of Computation, Vol. 38, No. 158, Jan 1982, pp. 339-374.

${ }^{16}$ Roe, P., "Fluctuations and Signals - A Framework for Numerical Evolution Problems," Numerical Methods for Fluid Dynamics, Dec 1982, pp. 219-257.

${ }^{17}$ Bultelle, M., Grassin, M., and Serre, D., "Unstable Godunov discrete profiles for steady shock waves," SIAM Journal on Numerical Analysis, Vol. 35, No. 6, Jan 1998, pp. 2272-2297.

${ }^{18}$ LeVeque, R., "Numerical Methods for Conservation Laws." Birkhauser Verlag AG, Jan 1994.

${ }^{19}$ Serre, D., "Remarks about the discrete profiles of shock waves," Mat. Contemp, Vol. 11, Jan 1996, pp. $153-170$.

${ }^{20}$ Dumbser, M., Moschetta, J., and Gressier, J., "A matrix stability analysis of the carbuncle phenomenon," Journal of Computational Physics, Vol. 197, No. 2, 2004, pp. 647-670.

${ }^{21}$ Temple, B., "Systems of conservation laws with invariant submanifolds," Transactions of the Americal Mathematical Society, Vol. 280, No. 2, Jan 1983, pp. 781-795.

${ }^{22}$ Bressan, A. and Jenssen, H., "Convergence of the Godunov scheme for straight line systems," Hyperbolic Problems: Theory, Numerics, Applications: Eighth International Conference in Magdeburg, Vol. 1, 2001, pp. 187-196.

${ }^{23}$ Myong, R. and Roe, P., "Shock waves and rarefaction waves in magnetohydrodynamics. Part 2. The MHD system," Journal of Plasma Physics, Jan 1997. 